\title{
Nanoimprinted strain-controlled elastomeric gratings for optical wavelength tuning
}

\author{
Yi-Chung Tung ${ }^{\text {a) }}$ and Katsuo Kurabayashi $i^{\text {b) }}$ \\ Department of Mechanical Engineering, University of Michigan, Ann Arbor, Michigan 48109
}

(Received 8 December 2004; accepted 1 March 2005; published online 14 April 2005)

\begin{abstract}
We demonstrate strain-controlled gratings made of an organic elastomer, polydimethylsiloxane (PDMS), which can achieve optical wavelength tuning by varying their spatial periods. The whole device structure presented in this work incorporates a nanoimprinted PDMS grating integrated with electrostatic microelectromechanical systems actuators on a silicon chip. The fabrication of the device combines polymer soft lithography, nanoimprint lithography, and silicon micromachining across multiscale dimensions ranging from a few hundred nanometers to a few millimeters. The fine tuning capability with fast dynamic response of our PDMS/silicon hybrid optical grating device makes it attractive for use in various micro-optical instruments. (c) 2005 American Institute of Physics. [DOI: 10.1063/1.1900923]
\end{abstract}

Optical wavelength tuning is one of the key functions for a large number of optical instruments. Wavelength tuningachieved by tunable lasers, tunable optical filters, and polychromators-has been exploited in a wide variety of applications, such as optical spectrometers, ${ }^{1,2}$ wavelength division multiplexing (WDM) (Ref. 3-6) photonics networks, and fiber Bragg grating (FBG) demodulators. ${ }^{7}$ Recently, a large amount of research has been devoted to develop highresolution, fast-response, and wide-range optical wavelength tuning devices to expand the capabilities of optical instruments. For instance, fast tunable filters have been recently incorporated in WDM photonics networks for highbandwidth optical communications. High-speed polychromators have been employed in real-time spectrometers for investigating transient phenomena. Microelectromechanical systems (MEMS) is one of the most promising technologies to provide the fast response required for these wavelength tuning applications. With the advancement of MEMS technology, several wavelength-tunable MEMS devices have been developed. Most of these devices operate based on Fabry-Perot cavity structures ${ }^{3-7}$ constructed with multiple dielectric thin films. However, they usually require complex structures, and their performance is often limited by a small tuning range and slow response. With rapidly increasing demands for an advanced fluorescence detection technology in many biological science applications, the capability of tuning a visible light wavelength at a high speed has become highly desirable. In addition, achieving fine wavelength tuning could further enable the real-time multiparameter fluorescence detection to simultaneously observe various biological activities.

In this letter, we report an optical MEMS device that can achieve fast-response and continuous wavelength tuning for visible light $(\lambda=400-800 \mathrm{~nm})$. We exploit diffraction gratings to spatially disperse optical spectrums with different wavelengths. Diffraction gratings can achieve high wavelength resolution with less attenuation ${ }^{8}$ and are suitable for realizing the aimed performance of the device. We provide

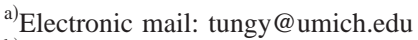

${ }^{b}$ Department of Electrical Engineering and Computer Science, University of Michigan, Ann Arbor, Michigan 48109.
}

the capability to dynamically reconfigure a surface pattern for the grating used in our device. The device employs an elastomeric material, polydimethylsiloxane (PDMS), as the grating material. PDMS is widely used in microfluidic applications ${ }^{9,10}$ and micro-optical components. ${ }^{11-13}$ Compared to silicon, which is commonly used in traditional micro-optical components, PDMS is optically transparent ( $\sim 92 \%$ transmission when accounting for loss at the air/ PDMS surfaces due to Fresnel reflection for $\lambda$ $=400-1000 \mathrm{~nm})$ and mechanically compliant $(\sim 750 \mathrm{kPa}$ Young's modulus and $\sim 40 \%$ maximum elongation). These unique material properties provide two technological advantages for our device; (1) Visible light can be transmitted through the grating component itself, facilitating the integration of the device with other optical components; and (2) varying the spatial period of the grating allows for continuously tuning the diffracted wavelength pattern. In our device, the spatial period of the PDMS grating component is varied under mechanical strain generated by electrostatic MEMS actuators. The unique functionality and performance of our on-chip device are made possible by combining the advantages of both silicon MEMS actuators and a mechanically flexible elastomeric micro-optical component.

Figure 1 shows the principle of the device operation used in this work. A transmission optical grating with a spatial period $a$ lies in the $x y$ plane, and a light source with a broad wavelength spectrum is incident to the $z$ direction. According to the grating equation, the wavelength of the

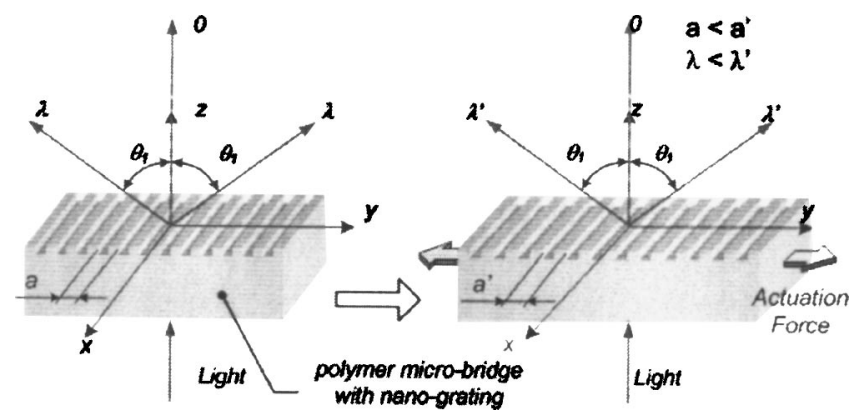

FIG. 1. Working principle of strain-controlled micro-optical grating. 


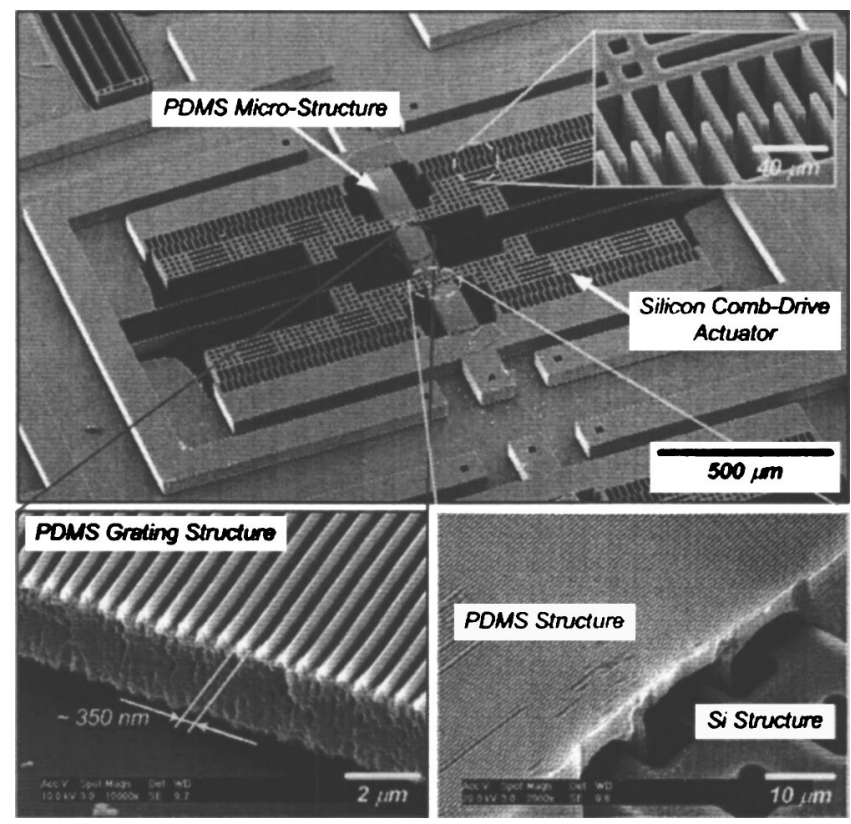

FIG. 2. SEM images of fabricated strain-controlled micro-optical grating. A PDMS grating microbridge with a nanoimprinted surface pattern is precisely attached onto silicon comb drives fabricated on a SOI chip.

first-order diffraction, $\lambda$, observed at a specific angle, $\theta_{1}$, can be given by

$$
\lambda=a \sin \theta_{1} .
$$

When introducing a mechanical strain to elongate the spatial period of the grating to $a^{\prime}$, the wavelength of the first-order diffraction at the same angle $\theta_{1}$ shifts to $\lambda^{\prime}$. The corresponding mechanical strain $\varepsilon$ can be defined as

$$
\varepsilon=\frac{a^{\prime}-a}{a}=\frac{a^{\prime}}{a}-1
$$

Then, the relation between the wavelength change $\Delta \lambda$ and the mechanical strain can be given by

$$
\Delta \lambda=\lambda^{\prime}-\lambda=a \varepsilon \sin \theta_{1}=\lambda \varepsilon .
$$

From Eq. (3), the wavelength shift of the first-order diffraction at the angle $\theta_{1}$ is linearly proportional to the mechanical strain introduced to the grating structure. Consequently, the peak wavelength of the diffracted light detected at a specific angle can be tuned by controlling the mechanical strain.

The strain-controlled micro-optical grating device reported in this study consists of a $600 \mu \mathrm{m} \times 100 \mu \mathrm{m}$ $\times 20 \mu \mathrm{m}$ PDMS microbridge that contains a nanoimprinted grating surface pattern with a $700 \mathrm{~nm}$ nominal pitch and two $50 \mu \mathrm{m}$ thick silicon comb drives fabricated on a silicon-oninsulator (SOI) wafer. The PDMS grating microbridge can be mechanically elongated by applying actuation voltage to the comb drives. Based on the fast electrostatic actuation mechanism, the comb drives enable the device to quickly change its diffraction pattern in response to a change in the applied actuation voltage.

To seamlessly integrate both the PDMS grating and the silicon actuators, we have developed a fabrication method named "multiscale soft lithographic lift off and grafting (MSSLLOG)." The MS-SLLOG process combines nanoimprint lithography, ${ }^{14}$ polymer soft lithography, ${ }^{15}$ and silicon micromachining to achieve a hierarchical device structure accom-

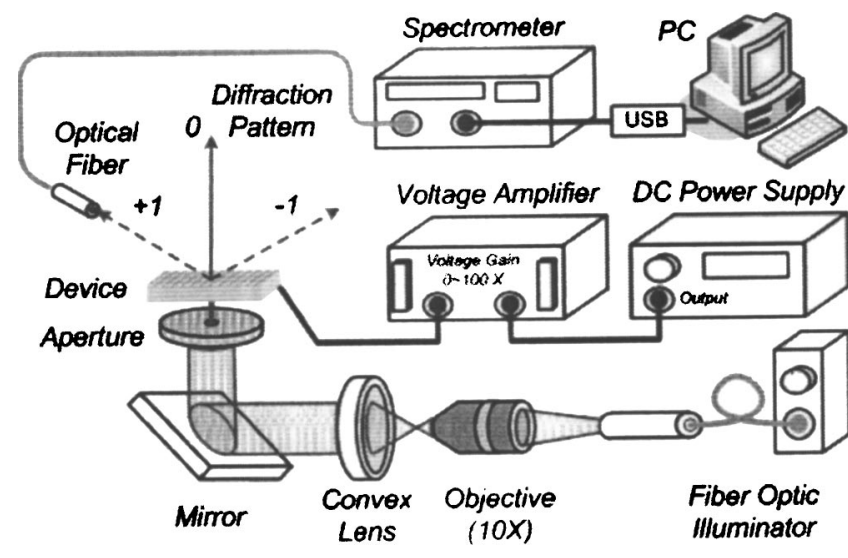

FIG. 3. Schematic of experimental setup for optical measurement to characterize strain-controlled grating device.

modating feature sizes ranging from hundreds of nanometers to submillimeters on a single chip. In this process, we used a soft lithography technique to construct the PDMS microbridge with the surface grating pattern. A liquid form of the PDMS precursor was placed, pressed, and cured between two micromolds containing the surface grating pattern and structural features of the PDMS microbridge. Here, we used a glass substrate coated with a photoresist layer with the grating pattern formed by nanoimprint lithography as the top mold in the soft lithography process. The other mold, which served as the bottom mold, was prepared by etching a mold pattern in a silicon substrate using deep reactive ion etching (DRIE). At the same time, we separately fabricated the silicon comb drives on a SOI wafer using silicon DRIE. After oxygen plasma surface treatment for both the PDMS and silicon surfaces, the cured PDMS microbridge structure was finally grafted onto these comb drives with $\pm 2 \mu \mathrm{m}$ alignment accuracy. This accurate alignment was made possible by introducing a fluidic microassembly technique. ${ }^{16}$ The resulting PDMS-silicon bond was strong enough to sustain the mechanical stress caused by the device operation. Figure 2 shows scanning electron microscopy (SEM) images of the fabricated grating device.

To demonstrate the wavelength tuning capability of our device, we measured the voltage-controlled variation of the optical spectrum of light diffracted in a specific direction by the PDMS grating. Figure 3 illustrates the experimental setup used for the device characterization. A fiber optic illuminator was used as a light source with a broad optical spectrum in the visible light range (about 300-700 nm). The light beam was focused into an objective lens $[10 \times$, numerical aperture $(\mathrm{NA})=0.25]$ and passed through a convex lens to minimize the divergence angle. The light beam was then guided by a mirror to reach the PDMS grating microbridge from the angle normal to the device back side. An aperture with a $1 \mathrm{~mm}$ diameter was placed in front of the position where the light beam was about to reach the grating. The aperture blocks the excessive light reaching outside the grating and serves to reduce optical noise. An optical fiber with a $100 \mu \mathrm{m}$ diameter core and NA=0.21 was placed close to the grating top surface with an offset from the right angle to collect the diffracted optical signal. Then, an optical spectrometer was employed to analyze the spectrum of the collected optical signal. 

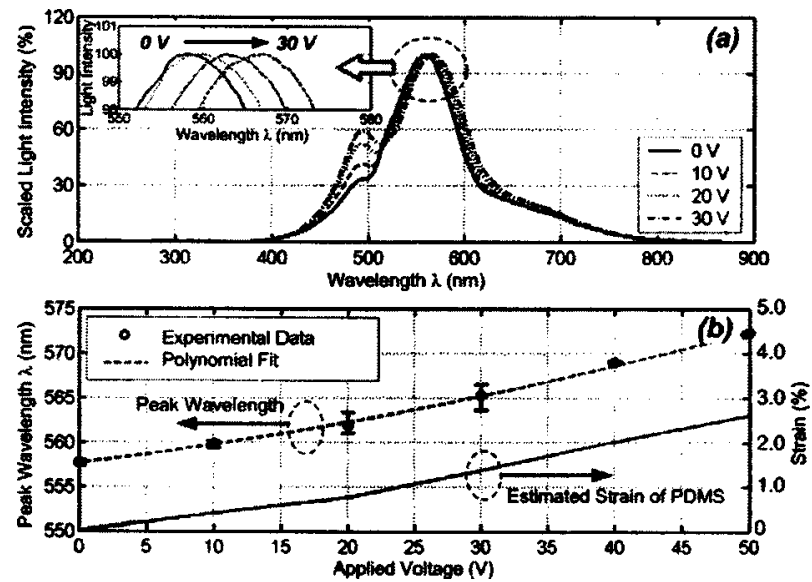

FIG. 4. Results of optical spectrum measurement. (a) Measured spectrum under various dc voltages applied to comb drives. (b) Peak wavelength shift and mechanical strain in PDMS microbridge at various actuation $\mathrm{dc}$ voltages.

Figure 4 shows the optical measurement results obtained using the spectrometer. Figure 4(a) shows the optical spectrums measured at various values of the dc voltage applied to actuate the comb drives. The figure shows a spectrum profile shifting toward longer wavelength with the increasing dc actuation voltage. Figure 4(b) plots the wavelength of the largest intensity (i.e., peak wavelength) in each spectrum versus the applied $\mathrm{dc}$ voltage, clearly showing the voltagecontrolled spectrum shifting demonstrated by our device. The peak wavelength continuously varies from about $557.7 \mathrm{~nm}$ to $572.3 \mathrm{~nm}$ as the dc actuation voltage increases from 0 to $50 \mathrm{~V}$. This variation is caused by the increase of the grating spatial period resulting from the mechanical strain introduced to the PDMS microbridge by the electrostatic force of the comb drive actuators. Figure 4(b) also plots the mechanical strain as a function of the dc actuation voltage, which is calculated from the measured wavelength shift and Eq. (3). At a $50 \mathrm{~V}$ dc actuation voltage, the mechanical strain introduced to the PDMS microbridge is about $2.5 \%$ for the tested device. In our device design, higher optical wavelength resolution can be easily achieved by reducing the optical fiber diameter and the NA for signal collection. In addition, the dynamic response of the device was characterized by measuring the temporal variation of the first-order diffraction angle for a monochromatic $\mathrm{He}-\mathrm{Ne}$ laser light source ( $30 \mathrm{~mW}$ power, $\lambda=632.8 \mathrm{~nm}$ ) at different ac actuation voltage signal frequencies. In this dynamic response characterization, the actuation bandwidth of the device was measured to be about $5 \mathrm{kHz}$.
In summary, we have developed a strain-controlled elastomeric micro-optical grating device with submicron-scale nanoimprinted polymer features. Its grating pitch can be mechanically varied under strain introduced by electrostatic MEMS actuators. This effect results in a voltage-controlled tuning of the grating device, which allows for tuning the wavelength of an optical setting in a well-controlled repeatable manner. Our study clearly demonstrated that the optical and mechanical properties of the elastomer, PDMS, permit the wavelength tuning capability desirable for various optical instrumentations that use visible light. The in-plane actuation scheme employed in the design eliminates the need for a mechanism to rotate the grating component in spectroscopy applications. With its demonstrated in-plane tuning capability, simple structural design, fast response, and transmission diffraction configuration, our device could be employed to develop unconventional micro-optical instruments, such as a real-time fluorescence spectrometer for lab-on-a-chip biological assay.

The authors thank Larry Cheng, Xing Chen, and Jay Guo at the University of Michigan for their support and technical inputs for the device fabrication. This work was supported by the National Science Foundation under CAREER Award and Contract No. ECS-0330963.

${ }^{1}$ H. L. Kung, S. R. Bhalotra, J. D. Mansell, D. A. B. Miller, and J. S. Jarris, Jr., IEEE J. Sel. Top. Quantum Electron. 8, 98 (2002).

${ }^{2}$ G. Lammel, S. Schweizer, S. Schiesser, and P. Renaud, J. Microelectromech. Syst. 11, 815 (2002).

${ }^{3}$ D. Hohlfeld, M. Epmeier, and H. Zappe, Proceeding of the 15th IEEE International Conference Microelectromechanical Systtem (IEEE, Piscataway, New Jersey, 2002), pp. 564-567.

${ }^{4}$ H. Halbritter, M. Aziz, F. Riemenschneider, and P. Meissner, Proc. SPIE 4945, 30 (2003).

${ }^{5}$ T. Amano, T. Hino, W. Janto, M. Arai, and F. Koyama, Jpn. J. Appl. Phys., Part 1 43, 157 (2004).

${ }^{6}$ M. Datta, M. W. Pruessner, D. P. Kelly, and R. Ghodssi, Solid-State Electron. 48, 1959 (2004).

${ }^{7}$ A. Unamuno, L. Li, and D. Uttamchandani, IEEE J. Sel. Top. Quantum Electron. 10, 598 (2004).

${ }^{8}$ D. M. Burns and V. M. Bright, Sens. Actuators, A 64, 7 (1998).

${ }^{9}$ D. Huh, Y.-C. Tung, H.-H. Wei, J. B. Grotberg, S. J. Skerlos, K. Kurabayashi, and S. Takayama, Biomed. Microdevices 4, 141 (2002).

${ }^{10}$ Y.-C. Tung, M. Zhang, C.-T. Lin, K. Kurabayashi, and S. J. Skerlos, Sens. Actuators B 98, 356 (2004).

${ }^{11}$ O. J. A. Schueller, D. C. Duffy, J. A. Rogers, S. T. Brittain, and G. M. Whitesides, Sens. Actuators, A 78, 149 (1998).

${ }^{12}$ K. Hoshino and I. Shimoyama, J. Micromech. Microeng. 13, 1 (2003).

${ }^{13}$ Y. Xie, X. Xu, Y. Hong, and S. Fu, Opt. Express 12, 3894 (2004).

${ }^{14}$ S. Y. Chou, P. R. Krauss, and P. J. Renstrom, J. Vac. Sci. Technol. B 14, 4129 (1996).

${ }^{15}$ Y. Xia and G. M. Whitesides, Annu. Rev. Mater. Sci. 28, 153 (1998).

${ }^{16}$ Y.-C. Tung and K. Kurabayashi, J. Microelectromech. Syst. (to be published in June 2005). 